\title{
PROFESIONALIZACIÓN PEDAGÓGICA DE LOS PROFESORES UNIVERSITARIOS: CASO UNIVERSIDAD LAICA ELOY ALFARO DE MANABÍ, EXTENSIÓN BAHÍA DE CARÁQUEZ
}

\author{
PEDAGOGICAL TRAINING FOR PROFESSORS: THE CASE OF ELOY \\ ALFARO UNIVERSITY AT BAHÍA DE CARÁQUEZ CAMPUS, MANABÍ
}

Arturo Santiago De la Rosa Villao, MS.c.

Docente Universidad Laica Eloy Alfaro de Manabí

Extensión Bahía de Caráquez, Ecuador

arturodlarosa@yahoo.es

Antonio Clarencio Guzmán Ramírez, Dr.C.

Docente Universidad Laica Eloy Alfaro de Manabí

Extensión Bahía de Caráquez, Ecuador

\section{RESUMEN}

Este trabajo hace un análisis de las limitaciones pedagógicas que tiene el profesor universitario frente a los requerimientos de la educación actual para la formación de profesionales y la necesaria profesionalización pedagógica del colectivo docente de la universidad, tendiente a elevar la calidad de la educación superior. Luego de describir la situación problemática se establecen los antecedentes investigativos, se hace un diseño teórico y metodológico, un sustento teórico sobre la profesionalización pedagógica del docente universitario, se realiza el diagnóstico de la situación actual, y se esboza un modelo y estrategia pedagógicos en base al vínculo docencia-investigación, para favorecer el desempeño pedagógico de los profesores de la Universidad Laica Eloy Alfaro de Manabí, extensión Bahía de Caráquez.

Palabras claves: Profesionalización pedagógica, desempeño pedagógico, docencia universitaria, vínculo docencia-investigación

\section{ABSTRACT}

This paper analyses pedagogical limitations that professors have against the requirements of current education for training of professionals and the necessary pedagogical professionalization of the faculty in order to raise quality at higher education. After describing the problem, research background is established, a theoretical and methodological design is carried out, a theoretical basis about pedagogical training, diagnosis of the current situation is undertaken, and a model and pedagogical strategy is outlined based on the linking of teaching and research in order to support pedagogical performance of teachers from Eloy Alfaro University at Campus Bahia de Caraquez.

Keywords: Pedagogical Training, Pedagogical Performance, Faculty, Teaching - Research link

\section{Introducción}

Recibido: diciembre de 2015 Aprobado: marzo de 2016

El propósito de la investigación es valorar la importancia de la profesionalización pedagógica de los docentes universitarios, con vista a fortalecer su desempeño para lograr una educación de calidad en la formación de los futuros profesionales.

La calidad de la educación es fundamental para el desarrollo de los pueblos, es necesario el compromiso de todos sus actores, de lo contrario no hay eficiencia de la educación ni desarrollo, es imperioso realizar una reestructuración en el sistema educativo con la finalidad de optimizarlo.

Existen investigaciones sobre esta problemática; unas la enfocan como formación inicial en el ámbito pedagógico, otras como formación continua; en el primer caso contempla la preparación de docentes en las Facultades de Educación, y en el segundo caso la preparación pedagógica en ejercicio de la docencia universitaria como necesidad para mejorar el desempeño con miras a mejorar la formación de los estudiantes. 
La investigación pretende aportar un modelo y estrategia pedagógica para favorecer el desempeño pedagógico de los profesores de la Universidad Laica Eloy Alfaro de Manabí (ULEAM), extensión Bahía de Caráquez, y sería importante si colegas de otras universidades lo adoptan en su gestión docente.

En las actuales circunstancias, el desempeño docente del profesor universitario no se circunscribe solamente a la docencia sino que además comprende gestión investigativa y de extensión que permita vinculación con la comunidad, la labor docente ha de ser también extra-clase. La investigación ligada estrechamente a la docencia da fundamentos científicos pedagógicos y de la profesión, por ende permite la superación permanente y consecuentemente mejora el desempeño pedagógico del profesor universitario, quien deberá convertir el aula de clase en un laboratorio de investigación para optimizar los procesos que la educación superior requiere.

\section{Desarrollo}

La situación problémica de la profesionalización pedagógica de los profesores universitarios

Organismos internacionales y el Ecuador están empeñados en provocar cambios radicales en la educación; la Organización de las Naciones Unidas tiene como uno de sus fines prioritarios mejorar la educación para elevar la calidad de vida de la población en los diversos países del mundo. Los Objetivos de Desarrollo del Milenio se lograrán en la medida que se impulse la educación; en el país, se han aprobado normas jurídicas para favorecer la preparación pedagógica y mejorar la formación de profesionales.

A partir de lo planteado por la conferencia mundial "La Educación Superior en el siglo XXI. Visión y Acción", de 1998, se determina entre las acciones prioritarias en el plano nacional: "Los Estados miembros...deberán formular políticas claras sobre los docentes de la educación superior", y en el plano de los sistemas e instituciones "la educación a lo largo de toda la vida exige que el personal docente actualice y mejore sus capacidades didácticas y sus métodos de enseñanza"[1].

La Declaración de la Conferencia Regional de la Educación Superior en América Latina y el Caribe, del 2008 en Cartagena, Colombia, establece respecto a la cobertura y modelos educativos e institucionales lo siguiente: "Hay que reconocer al cuerpo docente como actor fundamental del sistema educativo, garantizando su formación, ca- pacitación permanente, adecuadas condiciones laborales... y carrera profesional que permitan hacer efectiva la calidad en la enseñanza y la investigación"[2].

La conferencia mundial sobre Educación Superior del 2009, estipula como responsabilidad social respecto a la calidad de la educación superior la realización de investigaciones pedagógicas con el fin de mejorar las estrategias didácticas, cuyos resultados deben difundirse a través de las Tics y el acceso gratuito a la documentación científica, y los estados deben elaborar políticas y estrategias para garantizar las inversiones en investigación en sintonía con las necesidades de la sociedad, e impulsar las iniciativas de investigación conjunta entre docentes y estudiantes [3].

La exigencia y garantía del perfeccionamiento del docente universitario para mejorar la enseñanza y la investigación ha de traducirse en competencias, es decir su acción deberá ser el resultado sinérgico de conocimientos, habilidades y actitudes para el desarrollo de sus actividades en el ámbito profesional y consecuentemente en lo pedagógico y didáctico; la competencia constituye el conjunto de conocimientos, habilidades, actitudes y valores, combinados, coordinados e integrados en la acción, adquiridos a través de la experiencia profesional (formativa y no formativa), que permite al individuo resolver problemas específicos, de forma autónoma y flexible en contextos singulares [4].

Al referirse a las competencias se hace alusión a la profesión u oficio que ejecuta una persona, a la formación para la vida, a la capacidad productiva del individuo, de allí que se pueden clasificar las competencias bajo diversas ópticas, la más común en genéricas y específicas, básicas y profesionales; en el ámbito educativo se trata de competencias docentes, pedagógicas, didácticas, aspectos que coadyuvan a la formación pedagógica y al correspondiente desempeño del docente como producto de un proceso de profesionalización en el ámbito pedagógico.

Esta formación pedagógica comprende la capacidad que posee el docente para organizar y ejecutar el proceso de enseñanza aprendizaje, esto implica habilidades para planificar, determinar objetivos, preparar contenidos disciplinares, manejo de tecnologías, diseñar metodologías, organizar actividades, tutoriar, evaluar, trabajar en equipo, investigar, comunicación, labores de extensión respecto a la sociedad entre otros aspectos que se dan en los contextos de manifestación de la labor docente; en la medida que el profesor universitario adquiera formación pedagógica y se des- 
empeñe eficientemente se formará mejor al futuro profesional convirtiéndolo en un solucionador de problemas, pensador crítico y aprendiz permanente, para esto deberá emprender un proceso de profesionalización en el ámbito pedagógico; de esta forma la educación universitaria aportaría al desarrollo personal y social.

En el Ecuador, durante mucho tiempo no ha existido la decisión política de los gobiernos para implementar medidas que conduzcan a mejorar la educación, se la ha utilizado para satisfacer intereses de grupos en desmedro de la gran mayoría de la población; los últimos gobiernos han adoptados políticas que tienden a mejorar la calidad de la educación; el Plan Decenal de Educación del 2005, la Reforma Curricular de 1996 y su correspondiente actualización, la Constitución Política de la República del 2008, La Ley Orgánica de Educación Intercultural del 2010, la Ley Orgánica de Educación Superior del 2011, el Reglamento de Régimen Académico del 2013, constituyen la normativa jurídica que impulsan cambios en la formación de hombres y mujeres ecuatorianos.

El Estado ecuatoriano al tomar en consideración este ordenamiento jurídico, de orden externo e interno, está implementando cambios en todos los niveles educativos. La educación superior es objeto de profundas transformaciones en todos sus componentes curriculares; a nivel macro, el propósito es elevar la calidad de la educación; a nivel meso, las instituciones educativas deben ser pertinentes respecto a las necesidades y problemas sociales, y a nivel micro, los docentes deberán acceder a capacitación con la finalidad de optimizar el proceso de aprendizaje.

En las instituciones de educación superior los que ejercen la docencia son profesionales que se destacan en el ejercicio de su profesión y que se incorporan a la cátedra universitaria en consideración a sus méritos, en su mayoría son excelentes profesionales, pero no siempre poseen fundamentos pedagógicos para ser docentes universitarios; además, no existe institución para formar profesores para las universidades, esta tarea se convierte en responsabilidad de las propias universidades.

La docencia universitaria exige capacitación pedagógica y didáctica que tienda consecuentemente hacia la profesionalización, para que el proceso de aprendizaje se lleve a cabo ceñido a parámetros científicos para la formación de los futuros profesionales; la sociedad requiere de personas con formación suficiente para resolver los problemas que la afectan, éstas estarán preparadas en el sentido que los docentes pongan en práctica competencias pedagógicas y didácticas utilizando procesos y estrategias que permitan desarrollar habilidades, destrezas y actitudes en sus alumnos para enfrentar los retos de la vida social.

La carencia de conocimientos psicopedagógicos y didácticos, impide la instrumentación apropiada del proceso de enseñanza aprendizaje; por otra parte, se había descuidado la extensión como servicio a la comunidad y la investigación en la educación y sobre educación que el docente debería realizar como parte integrante de su labor; estos son problemas que afectan al colectivo docente de la Universidad Laica Eloy Alfaro de Manabí, extensión Bahía de Caráquez.

Al analizar los antecedentes de esta temática, los autores Manzo, J. (1999)[5], Sánchez, M. y García A. (2002)[6], García, G. (2005)[7], Marín, V. (2005)[8], López, L. (2005)[9],Espoch. (2008) [10],Christin, A.. (2010)[11], Ordaz, M. y Márquez, J. (2014)[12], Marcano, Y. (2014)[13], entre otros, han realizado investigaciones que enfocan diversos aspectos referentes a la formación inicial y profesionalización pedagógica del docente universitario.

No obstante en la Universidad Laica Eloy Alfaro de Manabí, extensión Bahía de Caráquez se aprecian vacíos en la labor pedagógica y didáctica de los docentes que afectan la calidad de formación pedagógica de los mismos, situación que se refleja en las siguientes evidencias empíricas:

- Docentes universitarios con escasos fundamentos pedagógicos al desarrollar sus clases, lo que no satisface los requerimientos de la formación de los estudiantes.

- Clases que no impactan positivamente en los futuros profesionales, a pesar de la importancia que puedan tener los contenidos.

- Desconocimiento de procesos didácticos que conllevan a apartarse de los componentes curriculares como las precisiones para la enseñanza aprendizaje, aplicación inadecuada de técnicas de aprendizaje.

- Poco interés de los docentes para prepararse pedagógicamente para desarrollar sus funciones profesionales.

- Docentes que muestran poco interés por conocer y participar de la sociedad y no influyen positivamente en los estudiantes.

- No se aprecia una preparación y trabajo metodológico en las disciplinas. 
- Existen docentes, principalmente a tiempo parcial, que no han recibido cursos de actualización en su especialidad en los últimos tres años.

- No existen documentos donde se proyecte la superación de los docentes según las necesidades de las disciplinas.

De esta situación problémica se aprecia una contradicción entre la insuficiente preparación pedagógica de los docentes de la Universidad Laica Eloy Alfaro de Manabí extensión Bahía de Caráquez y las exigencias de la sociedad ecuatoriana, en cuanto a la formación de profesionales; de esto adviene el siguiente problema científico a resolver:

¿Cómo perfeccionar el desempeño pedagógico en los docentes de la Universidad Laica Eloy Alfaro de Manabí, extensión Bahía de Caráquez?

El objeto de la investigación es la profesionalización pedagógica del docente universitario.

El objetivo de la investigación es la elaboración de una estrategia pedagógica sustentada en un modelo para perfeccionar el desempeño pedagógico de los docentes, desde el vínculo docencia-investigación, como parte de la superación permanente del docente de la Universidad Laica Eloy Alfaro de Manabí, extensión Bahía de Caráquez.

El campo de acción es el vínculo docencia - investigación.

Para el desarrollo de la investigación se plantea la siguiente hipótesis: Si se aplicara una estrategia pedagógica con énfasis en la vinculación docencia-investigación como parte de la superación permanente de los docentes universitarios, se podría perfeccionar el desempeño pedagógico de los docentes de la Universidad Laica Eloy Alfaro de Manabí, extensión Bahía de Caráquez.

Se realizaron las siguientes tareas científicas:

1. Fundamentación teórica y metodológica sobre la profesionalización pedagógica de los profesores universitarios.

2. Diagnóstico del estado actual de la profesionalización pedagógica de los docentes de la Universidad Laica Eloy Alfaro de Manabí, extensión Bahía de Caráquez.

3. Elaboración de un modelo pedagógico para perfeccionar el desempeño de los docentes de la Universidad Laica Eloy Alfaro de Manabí, extensión Bahía de Caráquez.
4. Diseño de estrategia a implementar para perfeccionar el desempeño pedagógico de los docentes de la Universidad Laica Eloy Alfaro de Manabí extensión Bahía de Caráquez.

5. Constatación de la utilidad de la estrategia aplicada para perfeccionar el desempeño pedagógico de los docentes de la Universidad Laica Eloy Alfaro de Manabí, extensión Bahía de Caráquez.

Respecto a la concepción metodológica, la investigación tiene un enfoque dialéctico materialista, ya que se realiza una valoración objetiva, tomando en consideración las contradicciones y particularidades que se presentan en la labor docente. De igual manera se tienen en cuenta el enfoque cuantitativo y el cualitativo, con predominio de este último.

Para el desarrollo de la investigación se aplicó métodos teóricos y empíricos.

Entre los métodos teóricos se utilizó el analítico-sintético, hipotético-deductivo, histórico-lógico, la modelación.

El método analítico-sintético permitió obtener un nivel de conocimiento más profundo sobre la formación pedagógica en los docentes de la Universidad Laica Eloy Alfaro de Manabí, extensión Bahía de Caráquez.

El método hipotético-deductivo para inferir conclusiones y predicciones sobre las variables: vínculo docencia-investigación y desempeño pedagógico, que se desarrollaron en la investigación.

El método histórico-lógico permitió valorar el desarrollo del proceso de profesionalización y desempeño pedagógico de los docentes de la extensión de Bahía de Caráquez y conocer los aspectos esenciales de dicho proceso.

El método de modelación sirvió de base para la estructuración de una estrategia pedagógica que vincula la docencia y la investigación tendiente a perfeccionar el desempeño pedagógico de los profesores universitarios.

Entre los métodos empíricos se utilizó la observación científica, la encuesta, la entrevista para recopilar información de campo sobre el desempeño pedagógico de los docentes de la extensión de Bahía de Caráquez

A través de la observación se obtuvo una percepción del problema en estudio: desempeño pedagógico del profesor en clase, uso de métodos, técnicas y recursos, si cumple con la finalidad de 
la evaluación, la integración de la investigación como parte substancial del proceso de enseñanza aprendizaje, entre otros aspectos.

La encuesta recogió información sobre el estado actual de la formación y desempeño pedagógico, conocimientos teóricos sobre las categorías didácticas.

La entrevista permitió obtener información de especialistas, de directivos sobre el empleo de métodos y técnicas que se privilegian en el proceso de clase y profundizar de manera particularizada en aspectos de interés pedagógico didáctico.

La población está constituida por los profesores de la Universidad Laica Eloy Alfaro de Manabí, extensión Bahía de Caráquez.; y la muestra conformada por los profesores de las carreras administrativas afines (Ingeniería Comercial, Auditoría, Marketing, Secretariado Ejecutivo, Hotelería y Turismo).

Tabla 1. Composición de la muestra:

\begin{tabular}{|l|c|c|}
\multicolumn{1}{c}{ Carreras } & Muestra & $\%$ \\
\hline Ingeniería Comercial & 4 & 17 \\
\hline Auditoría & 4 & 17 \\
\hline Secretariado Ejecutivo & 4 & 16 \\
\hline Hotelería y turismo & 5 & 21 \\
\hline Marketing & 7 & 29 \\
\hline Total & 24 & 100 \\
\hline
\end{tabular}

El tipo de muestreo es intencional no probabilístico. En la selección de la muestra se tomó en consideración las características de los sujetos especificadas en el planteamiento del problema y a la cantidad de profesores por carreras.

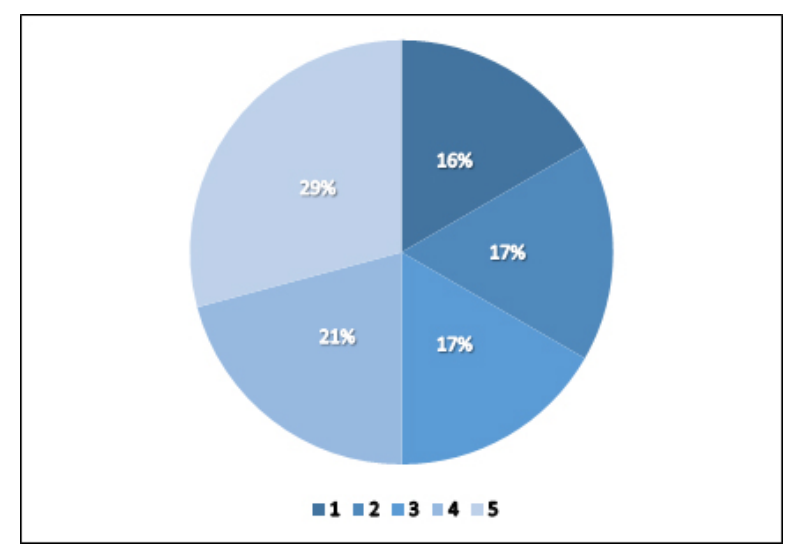

Figura 1. Composición de la muestra de profesores

El aporte teórico lo constituye un modelo pedagógico para perfeccionar el desempeño de los docentes universitarios, sustentado en los contextos de actuación y los criterios de desempeño que tiene como elemento rector la actividad investigativa del docente universitario.

El aporte práctico consiste en: una estrategia pedagógica para perfeccionar el desempeño de los docentes de la Universidad Laica Eloy Alfaro de Manabí, extensión de Bahía de Caráquez.

La novedad científica se encuentra en la dinámica del desempeño pedagógico del docente, que tiene como elemento rector la actividad investigativa del docente universitario integrado a su superación permanente.

La investigación se sintetiza en los siguientes aspectos:

\section{Acerca de la profesionalización pedagógica de los profesores universitarios}

La profesionalización es un proceso, que debe contribuir a la formación y desarrollo del modo de actuación profesional, desde una sólida comprensión del rol expresada en la lógica de la profesión y un contexto histórico determinado [14], caracterización que ha de considerarse tratándose de la profesionalización pedagógica del profesor universitario, que además aporta un conjunto de estrategias de regulación dentro de la enseñanza... que guardan estrecha relación con la función de la investigación y los discursos académicos... relaciones entre los campos políticos, ocupacionales y las ciencias educativas... logrando que los métodos de las disciplinas académicas organicen lo que los maestros y alumnos tienen que hacer [15]. El proceso docente educativo es, en sí mismo, un proceso de profesionalización del profesor universitario, en el que se articulan los componentes académico, laboral e investigativo, y adquiere dimensión axiológica al contribuir a la formación de valores en los sujetos inmersos en dicho proceso; el fin de la profesionalización del profesor universitario es elevar la calidad de la educación, formar profesionales competentes, capaces de insertarse en el mundo laboral a través del uso de la ciencia y la tecnología.

Factor importante que influye en la profesionalización pedagógica es la investigación, que constituye una vía efectiva para elevar el nivel profesional de los claustros académicos, al permitir una profundización mayor del proceso de enseñanza -aprendizaje, tener en cuenta su complejidad real y contribuir a perfeccionar el proceso educativo desde la instrucción [16].

En las actuales circunstancias, las acciones sistemáticas tendientes a la profesionalización peda- 
gógica del profesor universitario están determinadas por el nuevo rol que ha de adoptar el docente, deben estar encaminadas no sólo a la gestión pedagógica y didáctica sino además a la gestión de tutoría, de extensión y fundamentalmente, a las acciones investigativas en educación y sobre educación.

La profesionalización pedagógica demanda del profesor universitario como profesional de la educación un redimensionamiento en el desempeño de las funciones, en el que se constituya como un educador profesional para lograr la formación integral del estudiante universitario, adoptando una concepción amplia y compleja como orientador del estudiante en el proceso de construcción de conocimientos, desarrollo de habilidades y valores asociados a un desempeño profesional eficiente, ético y responsable [17], realizando sus acciones sustantivas de docencia, investigación y extensión, además de las relativas a su nuevo rol como tutor, orientador, mediador y solucionador de problemas, entre otras.

Se debe asumir la profesionalización pedagógica del docente universitario como potenciadora de los procesos formativos de los profesionales, un proceso complejo donde convergen varias dimensiones que se integran con carácter de sistema para determinar la competencia profesional; a saber:

a) Dimensión para la preparación pedagógica general, que exige del conocimiento y aplicación de conceptos pedagógicos para poseer cultura pedagógica general, que permita un enfoque sistémico del proceso de enseñanza aprendizaje.

b) Dimensión para la preparación psicodidáctica, a través de cursos de preparación didáctica, se conduzca acertadamente el proceso de enseñanza aprendizaje en las aulas universitarias.

c) Dimensión para la preparación metodológica de su especialidad, que el docente debe lograr en los diferentes niveles del trabajo metodológico como preparación pedagógica especializada.

d) Dimensión para la preparación socio-cultural, que mediará la superación permanente del docente universitario [18].

En estas cuatros dimensiones se integran los procesos sustantivos de docencia, investigación y extensión, que serán considerados parte de la profesionalización pedagógica del docente universitario, que como proceso va a mejorar la enseñanza aprendizaje en la universidad tendiente a optimizar la formación profesional de los estu- diantes y consecuentemente elevar la calidad de la educación superior.

\section{Diagnóstico del estado actual de la profesio- nalización pedagógica de los docentes de la Universidad Laica Eloy Alfaro de Manabí, ex- tensión Bahía de Caráquez}

El diagnóstico se realizó, en el primer semestre del año 2014, en base a indicadores establecidos a partir de la operacionalización de la variable: profesionalización pedagógica, a una muestra de 24 profesores, cuyos resultados se caracterizan a continuación:

De la encuesta realizada a los profesores de la ULEAM Bahía, se observa que los docentes vienen laborando en la universidad: el $46 \%$ entre uno a tres años, el $25 \%$ entre cuatro y seis años, el $25 \%$ entre siete y nueve años, y el $4 \%$ diez años o más; el $67 \%$ labora a tiempo completo, y el $33 \%$ a tiempo parcial; el $96 \%$ de los investigados posee títulos de pregrado en diversas carreras ajenas a la educación, el $4 \%$ tiene título pedagógico; el $13 \%$ posee posgrados afines a sus carreras, el $45 \%$ tiene un diplomado relacionado a educación, y el $42 \%$ no posee título de posgrado; en los últimos cinco años, el $46 \%$ ha realizado cursos y seminarios relacionados a su profesión, y el 54 \% no lo ha hecho; respecto a capacitación docente, el 50 \% ha realizado un curso mientras que el otro $50 \%$ no; el $67 \%$ no conoce de teorías pedagógicas y categorías didácticas; el $67 \%$ no ha realizado investigaciones de carácter pedagógico; el 12,5 ha realizado investigaciones formativas, y el $87,5 \%$ no ha realizado; el 100 $\%$ no ha realizado investigaciones generativas; el $75 \%$ envía muy frecuentemente investigaciones a sus estudiantes, y el $25 \%$ lo hace de manera frecuente.

Esta información se complementa con los datos, que a través de una encuesta, proporcionaron los estudiantes. La misma cuenta con 6 preguntas y utiliza una escala de rango cuatro, que establece las siguientes categorías:

$\begin{array}{ll}\text { Nunca: } & 1 \\ \text { Poco frecuente: } & 2 \\ \text { Frecuentemente: } & 3 \\ \text { Siempre: } & 4\end{array}$

Se aplica un procedimiento de muestreo probabilístico debido a que la población de estudiantes está censada, en proporción de la cantidad de estudiantes por carreras. El cálculo del tamaño de muestra se realiza utilizando la siguiente expresión: 


$$
n=\frac{N^{*} K^{2} P * Q}{e^{2}(N-1)+K^{2} * P * Q}
$$

Donde:

n: Tamaño de muestra

$\mathrm{N}$ : Tamaño de la población

$P$ y $Q$ : valores de probabilidades relacionadas con

la ocurrencia o no del evento.

El valor de $\mathrm{K}$ es constante e igual a 1,96 siempre que se trabaje con un nivel de confianza del $95 \%$. Los valores de $P=Q=0,5$, ya que no se tiene información alguna al respecto.

Población $\mathrm{N}=510$

$\mathrm{P}=\mathrm{Q}=0.5$

$\mathrm{K}=1.96$

$\mathrm{e}=0.05$

$\mathrm{n}$ total $=225$, se aplican 225 encuestas a estudiantes.

La composición de la muestra es la siguiente

Tabla 2. Composición de la muestra estudiantes

\begin{tabular}{|l|c|c|}
\multicolumn{1}{c}{ Carreras } & Muestra & $\%$ \\
\hline Ingeniería Comercial & 38 & 17 \\
\hline Auditoría & 38 & 17 \\
\hline Secretariado Ejecutivo & 36 & 16 \\
\hline Hotelería y turismo & 48 & 21 \\
\hline Marketing & 65 & 29 \\
\hline Total & 225 & 100 \\
\hline
\end{tabular}

Figura 2. Composición de la muestra estudiantes

Tabla 3. Resultados de la encuesta a estudiantes

\begin{tabular}{|l|c|c|c|}
\hline \multicolumn{1}{|c}{ Preguntas } & \multicolumn{1}{c|}{ Media } & \multicolumn{1}{c|}{ Median } & \multicolumn{1}{c|}{ Moda } \\
\hline $\begin{array}{l}\text { La metodología satisface sus } \\
\text { necesidades de aprendizaje }\end{array}$ & 1,36 & 1.5 & 2 \\
\hline $\begin{array}{l}\text { Preparación de los estudian- } \\
\text { tes para realizar investigacio- } \\
\text { nes }\end{array}$ & 1.71 & 1.5 & 2 \\
\hline $\begin{array}{l}\text { Preparación de los estudian- } \\
\text { tes para realizar trabajos in- } \\
\text { dependientes }\end{array}$ & 1,28 & 1.5 & 2 \\
\hline $\begin{array}{l}\text { Preparación pedagógica y } \\
\text { metodológica. }\end{array}$ & 1,68 & 1.5 & 2 \\
\hline Formación integral & 1,66 & 1.5 & 2 \\
\hline Satisfacción general & 2,92 & 1.5 & 2 \\
\hline
\end{tabular}

Los resultados generales muestran que el $60 \%$ de los estudiantes encuestados manifiestan que la metodología que utiliza el profesor de manera poco frecuente satisface sus necesidades de aprendizaje

El $47 \%$ consideran que es poco frecuente que los profesores preparan a los estudiantes para que realicen investigaciones.

El $57 \%$ dice que es poco frecuente que los profesores preparan a los estudiantes para que realicen trabajos independientes.

El $60 \%$ considera que es poco frecuente que los docentes demuestren en clase preparación pedagógica y metodológica.

El $57 \%$ valora que es poco frecuente que sus profesores contribuyen a su formación integral.

Estos resultados se hacen más críticos en las carreras de Secretariado Ejecutivo, Auditoría e Ingeniería Comercial, en las carreras de Marketing y Hotelería y Turismo presentan valores desfavorables, pero superiores a las de dichas carreras En general se puede inferir a partir de la opinión de los estudiantes que el $57 \%$ de ellos considera que sus profesores no los preparan adecuadamente, ni integralmente y no están satisfechos con sus docentes.

Para validar la encuesta utilizada a estudiantes se le realizan las pruebas de fiabilidad y validez del instrumento utilizado, mediante el paquete estadístico SPSS. Versión 21. Los resultados obtenidos fueron:

Estadísticos de fiabilidad
Alfa de Cronbach $\quad$ N de elementos
\begin{tabular}{|l|l|}
\hline, 915 & 6 \\
\hline
\end{tabular}

El valor del coeficiente Alfa de Cronbach es superior a 0.80 lo que indica que el instrumento utilizado está libre de errores aleatorios y que las mediciones son estables y confiables.

En cuanto a la validez del instrumento, para ello se calcula el coeficiente de correlación lineal R2. Los resultados fueron:

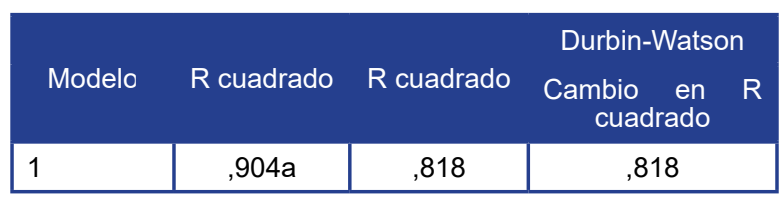

El valor de $\mathrm{R}^{2}$.es superior a 0.7 , lo que indica que la encuesta utilizada está exenta de errores aleatorios y sistemáticos y que realmente está midiendo lo que se pretende; por lo tanto es válida 
Se realizó observación durante el desarrollo de clase para constatar la implementación de procesos didácticos y acciones investigativas: en el80\% de las clases observadas no se evidencia organización de las categorías didácticas en el proceso; en el $80 \%$ no se evidencia el nexo de las actividades de aprendizaje del tema estudiado con acciones que fomenten la investigación científica, ni se prioriza, ni se demuestra el uso de estrategias investigativas en el desarrollo de la clase.

Además, se realizó entrevista a directivos de la ULEAM Bahía para evidenciar la profesionalización pedagógica del colectivo docente: no existen programas de capacitación en la extensión para lograr la preparación pedagógica de los docentes; el desempeño de los docentes se valora como nivel medio, muy pocos cumplen a cabalidad; los métodos utilizados por los docentes no favorecen la actividad creadora de los estudiantes; existe poca formación permanente de los docentes de la extensión en aspectos relativos a su profesión y en lo pedagógico; no hay investigación científica en el ámbito de la profesión y en lo pedagógico; $y$, para mejorar la profesionalización pedagógica de los docentes sugieren incentivar la lectura en el área profesional y en educación; realizar congresos, cursos, seminarios talleres orientados a investigación, tecnología, pedagogía y didáctica.

\section{Modelo pedagógico para perfeccionar el des- empeño de los docentes de la Universidad Lai- ca Eloy Alfaro de Manabí extensión Bahía de Caráquez}

La complejidad de la sociedad deriva en problemas de toda índole y espera respuestas de sus instituciones para satisfacer sus necesidades y resolver dichos problemas, y es precisamente la universidad, como matriz del conocimiento, la encargada de emprender estas acciones cumpliendo con el encargo social a ella encomendada: la formación de profesionales; para ello necesita que todos quienes integran la universidad optimicen sus esfuerzos con la finalidad de elevar la calidad de la educación y consecuentemente coadyuvar a la formación de profesionales con las competencias necesarias acorde a los requerimientos de la sociedad, para estos efectos es indispensable la preparación del docente universitario.

Es evidente que el profesor universitario tiene limitada preparación profesional en lo pedagógico; por lo general, está preparado profesionalmente, en alguna disciplina pero no ha sido formado para ser profesor universitario, presentándose cierta situación problémica frente a la necesidad de orientar los procesos de formación de los estudiantes; para esto se requiere no solamente voluntad y optimismo sino que además se demanda una sólida preparación profesional en su carrera, y por otra parte capacitación en el orden pedagógico y didáctico para implementar de manera eficiente el proceso de aprendizaje; capacitación que el docente lo adquirirá a través de autopreparación, educación continua o procesos que la propia universidad deberá emprender, de tal manera que en la universidad se enseñe a los profesores a educar, para que los estudiantes aprendan a aprender [19].

Es menester, por lo tanto, que el profesor universitario acceda a un proceso de profesionalización pedagógica que le permitan dominio de las ciencias pedagógica y didáctica, con sus leyes, principios y categorías que le darán fundamentos para instrumentar adecuadamente el proceso de enseñanza aprendizaje, considerando el nuevo rol del docente que se resume en las gestiones de docencia, investigación y extensión.

El ejercicio del docente universitario en la actualidad se ha diversificado y está ligado de manera esencial a la investigación científica en la profesión y pedagógica, no se concibe docencia sin investigación, este vínculo le va a proporcionar los saberes necesarios para mejorar su gestión ligada a la superación permanente en pedagogía general, psico-didáctica y metodología particular, preparación profesional y en lo sociocultural; dimensiones que conllevan al desempeño pedagógico integral y contextualizado del docente como resultado de la profesionalización pedagógica potenciadora de los procesos formativos de los profesionales, que es el producto que la sociedad espera de la universidad.

En el desempeño pedagógico, el profesor universitario es un agente de cambio, su tarea no es sólo transmitir información, sino realizar un proceso de enseñanza que genere aprendizajes de forma significativa y contextualizada para lograr mayor rendimiento académico y desarrollo integral del estudiante[20]; esto conlleva al profesor a mejorar su práctica docente.

La formación profesional de los estudiantes, que constituye la misión de las instituciones de educación superior, depende en gran medida del desempeño pedagógico de sus profesores; tal desempeño está relacionado con los conocimientos, habilidades y actitudes que deben poseer los profesionales de la educación para asegurar que los estudiantes alcancen los aprendizajes deseados [21] determinados por los estándares de calidad de la educación.

El desempeño pedagógico del profesor universi- 
tario refleja una valoración positiva hacia la profesionalización docente como medio para mejorar la calidad de la educación superior, de tal manera que el profesional que egrese de la universidad este dotado de todas las herramientas cognitivas, procedimentales y axiológicas como resultado de la labor docente, que le permitan resolver los problemas sociales y coadyuvar al desarrollo social; de esta manera la formación de profesionales se constituye, en un proceso desarrollador, interactivo, mediado por la comunicación como nexo de la socialización del individuo, que posibilite la apropiación activa de la cultura, construya sus propios conocimientos, desarrolle autonomía, autodeterminación y reflexión dialéctica [22], que favorezca la formación de valores y la responsabilidad social, que tome en cuenta las potencialidades y necesidades de los estudiantes.

En el siguiente esquema se ilustra el modelo pedagógico.

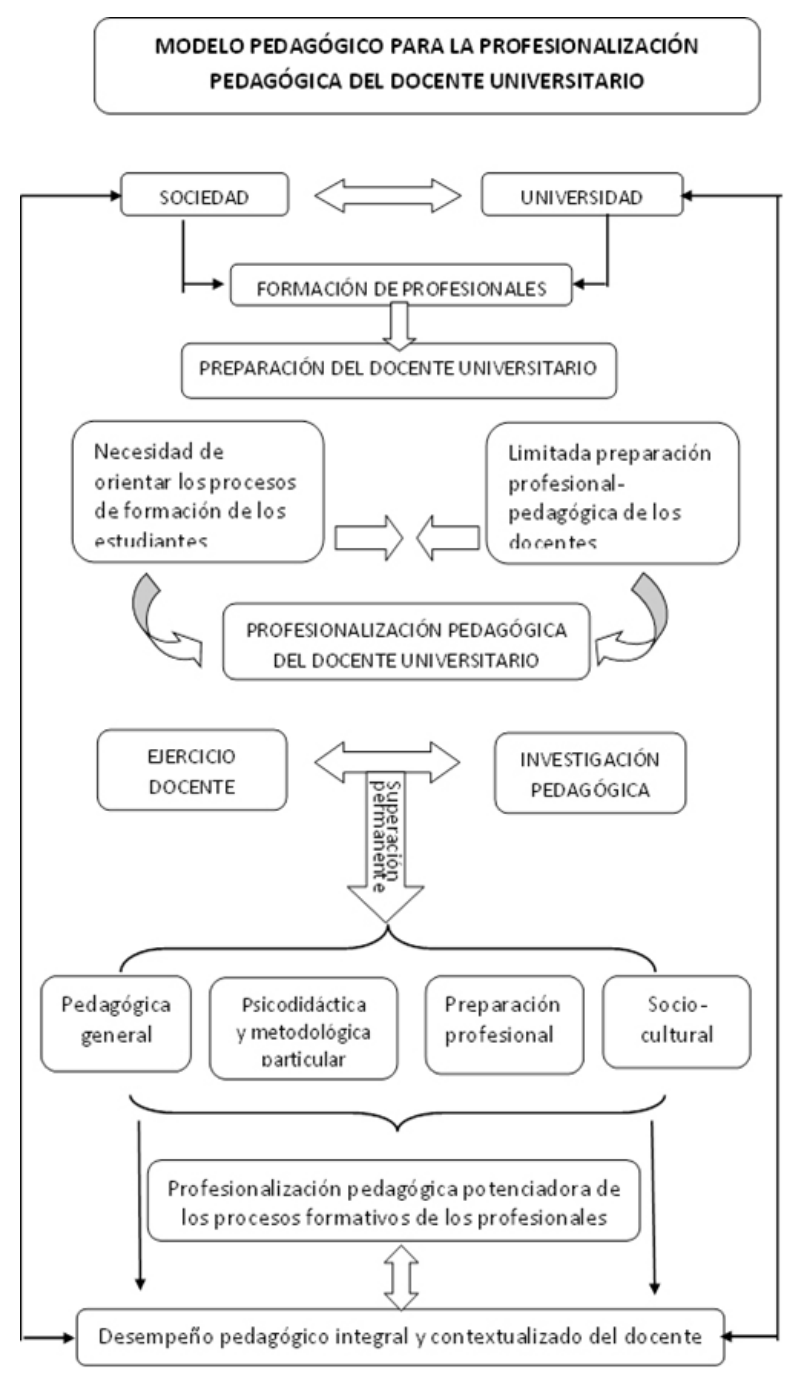

Figura 3. Esquema del modelo pedagógico
Estrategia a implementar para perfeccionar el desempeño pedagógico de los docentes de la Universidad Laica Eloy Alfaro de Manabí extensión Bahía de Caráquez

A continuación se bosqueja la estrategia pedagógica a implementar

ESTRATEGIA PARA PERFECCIONAR EL OESEMPEÑO DOCENTE
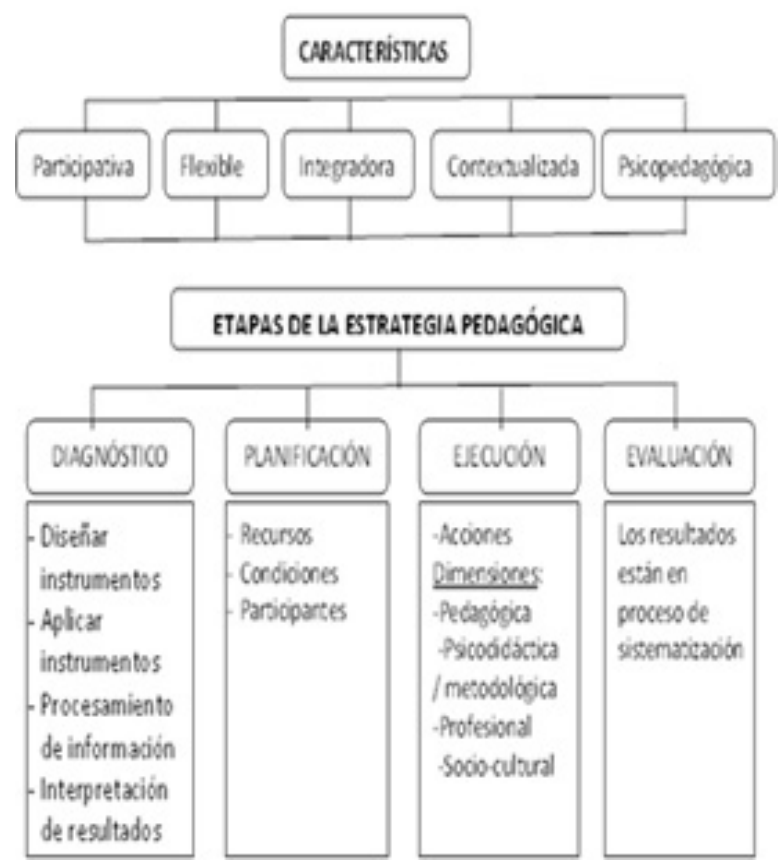

Figura 4. Esquema de la estrategia pedagógica

Luego del diagnóstico y la estructuración del modelo, en la ULEAM, extensión Bahía de Caráquez se implementó la estrategia pedagógica consistente en cursos, seminarios y talleres en el ámbito pedagógico e investigativo.

Se llevó a efecto un plan de capacitación que contempla diseño y elaboración de investigaciones, docencia e investigación pedagógica, investigación en el aula de clase, redacción científica, estadística aplicada a la investigación, y evaluación de proyectos.

En el proceso de capacitación, el $60 \%$ de los profesores poseen maestrías, 8 \%realizan doctorados en las repúblicas de Cuba y Perú. El 50 \% han emprendido acciones investigativas vinculadas a la profesión y la pedagogía, y el 50 \% ha realizado publicaciones científicas, en diversas revistas indexadas.

Las evaluaciones de desempeño realizadas semestralmente desde el año 2013 al 2015 han evidenciado una mejora continua del desempeño pedagógico de los profesores, lo que ha permitido cumplir con los indicadores de evaluación estable- 
cidos por el Consejo de Evaluación, Acreditación y Aseguramiento de la Calidad de la Educación Superior, alcanzando la acreditación de la extensión universitaria de la ULEAM en Bahía de Caráquez a través de la resolución NO. 546-CEAACES-SE-16.2015, del 7 de agosto del 2015.

\section{Conclusiones}

La información permitió hacer un diagnóstico que refleja el estado actual de la situación problémica que afecta al colectivo docente de la Universidad Laica Eloy Alfaro de Manabí, extensión Bahía de Caráquez, y en respuesta la universidad está emprendiendo acciones con miras a mejorar el proceso docente educativo.

Los presupuestos teóricos permiten tener una visión científica del problema y planificar actividades y estrategias para favorecer el desempeño pedagógico de los docentes.

\section{Recomendaciones}

El modelo pedagógico favorecerá el desempeño de los profesores universitarios con la finalidad de mejorar los procesos de formación de los profesionales y consecuentemente elevar la calidad de la educación superior.

La estrategia pedagógica se constituye en el instrumento que dinamiza la gestión del profesor universitario, que de acuerdo a las exigencias actuales comprende docencia, investigación, labores de extensión con respecto a la comunidad, tutoría, orientación, desarrollo de habilidades de comunicación y manejo de tecnología, diseño de metodologías y estrategias, trabajo en equipo, entre otros aspectos que se dan en los contextos de actuación del docente.

El profesor universitario ha de emprender procesos de capacitación en su profesión y en lo pedagógico, tendiente a profesionalizar la labor docente con la finalidad de mejorar el proceso de enseñanza aprendizaje

\section{Referencias Bibliográficas}

[1] Unesco. Conferencia Mundial la educación superior en el siglo XXI. Visión y acción. Informe final. París. (1988).

[2] Unesco. Conferencia Regional de la Educación Superior en América Latina y el Caribe (CRES) Declaraciones y plan de acción. Perfiles Educativos, vol. XXXI, núm. 125, 2009, pp. 90-108. Redalyc. org. (2008).
[3] Unesco. Conferencia Mundial sobre la Educación Superior. La nueva dinámica de la educación superior y la investigación para el cambio social y el desarrollo. París. (2009).

[4]Tejada, José. Competencias docentes. Profesorado. Revista de currículum y formación del profesorado. Vol. $13 \mathrm{~N}^{\circ}$ 2. (2009).

[5]Manso Martínez, José Ma. Profesionalización pedagógica del profesorado universitario. Cáceres. España Revista interuniversitaria de formación del profesorado. № 34 Enero/abril 1999, pp. 319-328 (1999).

[6] Sánchez, María y Ana García. Formación y profesionalización docente del profesorado universitario. Universidad de Salamanca. Revista de Investigación Educativa. Vol. 20, nº 1, págs. 153171 (2002).

[7] García, Gilberto Curso 8. La práctica pedagógica y la profesionalidad del docente. Pedagogía 2005. Unesco. Iplac. La Habana. (2005)

[8]Marín, Verónica. Desarrollo profesional del docente universitario a debate: Factores que lo determinan. Córdova. Argentina. (2005).

[9] López, Lutgarda y Coralia Pérez. La investigación como eje de articulación en los currículos para la formación de profesores en las condiciones actuales. Universidad de Cienfuegos. Cuba. (2005).

[10]Espoch. Sistema de profesionalización docente de la Escuela superior politécnica de Chimborazo. (2008).

[11] Christin, Alberto Tendencias en la Profesionalización de los Docentes REDDOLAC.www.reddolac.org/profiles/blogs/tendencias-en-la-1. (2010)

[12] Ordaz Hernández, Maira y Juan Márquez M. Capacitación del profesor universitario para la orientación psicopedagógica. Universidad de Piñar del Río. Iceces. Cuba. Revista Iberoamericana de Educación. № 62/2 2014Marcano. (2014).

[13]Marcano Fierro, Ybelise. La Profesionalización y el Desempeño Profesional Pedagógico del Docente Universitario: Una visión Transdisciplinaria. Red Iberoamericana de docentes. (2014).

[14] Addine Fernández, F. yA. Blanco Pérez, La profesionalización del maestro desde sus funciones fundamentales. Algunos aportes para su comprensión. Dirección de Ciencia y Técnica del MINED. La Habana. (2002). 
[15]León Hernández, Vicente E. y Jorge Herrera Fuentes. Una visión de la profesionalización como categoría de las ciencias de la educación. Cuadernos de Educación y Desarrollo. Vol. 2, № 13. Año 2010. Piñar del Río. (2010).

[16] Ortíz Torres, Emilio y María de los Ángeles Mariño Sánchez. La profesionalización del docente universitario a través de la investigación didáctica desde un enfoque interdisciplinar con la psicología. Universidad de Holguín. Cuba. Revista Iberoamericana de Educación N ${ }^{\circ} 35 / 6$ (ISSN: 16815653). (2005).

[17] Mendoza Jacomino, Carlos, Artiles Olivera Iliana, Torres Valladares, Javier. Ponencia: La formación profesional y la profesionalización del profesor universitario. Retos para la calidad educativa. Universidad Central Marta Abreu de las Villas. Cuba. Congresos WEB. Plataforma de E-Congress de Redcyt. (2011).

[18]Guzmán Ramírez, Antonio. Ponencia: La profesionalización pedagógica de los docentes universitarios: Pertinencia universidad-sociedad. Universidad Oscar Lucero Moya. Ceces. Holguín, Cuba. (2013).

[19] Cáceres, Maritza. La formación pedagógica de los profesores universitarios, una propuesta en el proceso de profesionalización del docente. Universidad de Cienfuegos. Cuba. (2003). www. bibliociencias.cu/gsdl/collect/revistas/index

[20] Monja, María Isabel. Desempeño docente. Calidad educativa desde la perspectiva del desempeño docente. Blog WordPress.com: http:// mariaisabelmonja.wordpress.com/2012/02/06/ desempeno-docente. (2012).

[21]Ministerio de Educación del Ecuador. Estándares de calidad de la educación. Quito. (2011).

[22]Ortíz, Alexander. Aprendizaje desarrollador: Una estrategia pedagógica para educar instruyendo. Centro de Estudios Pedagógicos y Didácticos. Barranquilla. (2005). 